\title{
Numerical Prediction and Experimental Validation of Multiple Phosphor White LED Spectrum
}

\author{
Jeffery C. C. LO $^{1 *}$, S. W. Ricky LEE ${ }^{1,2,3}$, Xungao GUO ${ }^{2}$, Huishan ZHAO ${ }^{2}$ \\ ${ }^{I}$ Center for Advanced Microsystems Packaging \\ ${ }^{2}$ HKUST LED-FPD Technology R\&D Center at Foshan \\ ${ }^{3}$ Department of Mechanical and Aerospace Engineering \\ Hong Kong University of Science \& Technology \\ Clear Water Bay, Kowloon, Hong Kong \\ *Tel: +852-23588356,Fax: +852-23588357, e-mail:jefflo@ust.hk
}

\begin{abstract}
Conventional phosphor converted white light LEDs utilize yellow phosphor to convert the blue light emitted from the chip to yellow light. Although this can generate white light with various correlated color temperatures (CCT), the color rendering properties are generally poor as the spectrum of the LEDs, as compared with the reference light source, is not broad enough. The red light output is low in particular. This limitation is caused by the emission spectrum of yellow phosphor. To improve the color rendering properties, multiple phosphors with different emission spectra should be used. For instance, a certain amount of red or orange phosphors may be used to mix with yellow phosphor to increase the output in the red region. In this paper, a numerical model is proposed to predict the emission spectra of LEDs with multiple phosphors. The excitation and emission spectra of various phosphors are measured and input to the model. The mixing ratio and reabsorption between phosphors have also been taken into consideration. White light LEDs with multiple phosphors are fabricated. The spectra are measured and compared with the modeling results. It is found that the proposed model can estimate the emission spectra of LEDs with multiple phosphors with a high degree of accuracy.
\end{abstract}

Keywords-spectrum prediction, multiple phosphors, color rendering

\section{INTRODUCTION}

When selecting light sources for different applications, there are a lot of considerations, such as lumen output, efficiency, color temperature, etc. In general lighting, the color rendering capability of the light source is equally important [1]. A light source with a good color rendering capability can show the color of the illuminated object in greater accuracy and make it appear natural. There are various metrics to quantify the color rendering performance of a light source, such as color rendering index (CRI) [2], color quality scale (CQS) [3], fidelity index (Rf), gamut index $(\mathrm{Rg})$ [4], etc.

In calculating the color rendering properties, the test light source is compared with a reference light source. The reference light source is usually selected from incandescent light bulb (black body radiation) or CIE D series (day light), depending on the color temperature of the test light source [5]. The spectra of the reference light sources are very broad. These light sources can reveal a full range of colors without distortion, hence making the color of the illuminated object appear natural.

Conventional phosphor converted LEDs utilize yellow phosphors to convert the blue light emitted from the chip to yellow light. The emission peak of conventional yellow phosphor is usually at around $550-560 \mathrm{~nm}$. This is close to the peak of the CIE spectral luminous efficiency function of photopic vision which is at $555 \mathrm{~nm}$. This leads to a higher luminous output, making the LED package look "brighter" to observers. Although white light with various CCT can be achieved [6-10], the color rendering properties are normally low. It is because the spectrum of the LED is not broad enough when compared with the reference light source as illustrated in Fig. 1. This limitation is due to the emission spectrum of yellow phosphor which has a low red light output in particular. In order to improve the color rendering properties, multiple phosphors with different emission spectra are used [11-13]. For instance, a portion of red or orange phosphors are used to mix with yellow phosphor to increase the output in the red region [14-18].

Luminous flux, CCT, color rendering index, among others, are key parameters to describe the optical performance of a LED package. These parameters are calculated from the LED spectrum. An accurate spectrum prediction model is a useful tool at the package design stage [19]. Sun et al. proposed different models to predict the spectrum of a white LED with multiple phosphors. In their calculation, only the phosphors emission spectra were considered [20]. However, in LEDs with multiple phosphors, the excitation spectrum of red phosphor overlaps with the emission spectrum of yellow phosphor. A portion of

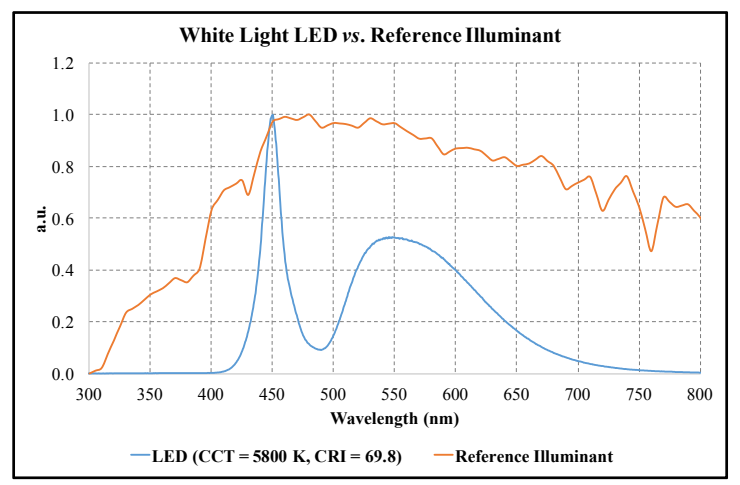

Fig. 1 White Light LED Spectrum vs Reference Illuminant Spectrum 
yellow light will be absorbed by red phosphor and then turned into red light. This reabsorption phenomenon makes the calculation of the final spectrum difficult. The final spectrum cannot be estimated by superposition of phosphor emission spectra.

In this paper, a numerical model is proposed to predict the emission spectrum of white LEDs with multiple phosphors. The excitation and emission spectra of each phosphor are measured and input to the model. The proposed model has also taken the mixing weight ratio of phosphors, the reabsorption and the reemission phenomenon into consideration. White LEDs with multiple phosphors are fabricated. The spectra are measured and compared with the calculated results. It is found that the proposed model can predict the emission spectra of LEDs with multiple phosphors in a high degree of accuracy. The CIE 1931 $x y$ chromaticity coordinate error is within $+/-0.003$.

\section{PHOSPHOR CHARACTERIZATION}

The excitation and emission spectra of phosphors materials are important parameters in the proposed prediction model. In this study, two red phosphors (namely R1 and R2) and two yellow phosphors (namely Y1 and Y2) are used. The spectra can be obtained by a spectrofluormeter. The excitation spectrum records the peak emission intensity while the phosphor material is excited by the corresponding monochromatic light beam. This provides a guideline to select a proper LED chip to match with the phosphor material. If the peak wavelength of the LED chip is close to the phosphor excitation peak wavelength, a high emission intensity will be obtained. Fig. 2 shows the excitation spectra of Y1 and R1. The excitation peak wavelength of yellow phosphor is usually at the blue light region, which is close to the peak wavelengths of most LED chips. On the other hand, red phosphor has a border excitation band.

An absorption spectrum is used to describe the absorption phenomenon of the phosphor material. It provides more accurate prediction results but it is difficult to measure. To a certain extend, the excitation spectrum also includes phosphor absorption information. A high emission level is usually associated with a high absorption level. For simplicity, the normalized excitation spectrum is used in this study to calculate the amount of light being absorbed by the phosphor material at different wavelengths.

The emission spectrum is another important parameter in the calculation of the spectra of multiple phosphors. The emission spectrum defines the light emitted behavior of the phosphor material. The color of the phosphor material is associated with its emission spectrum. Fig. 3 shows the emission spectra of Y1 and R1 used in this study. The emission bands of most phosphor materials are narrow. This usually leads to a poor color rendering property of phosphor converted white LEDs.

The emission spectrum is determined by the energy band gap of the phosphor material. The normalized emission spectrum is independent of the wavelength of the incident light. Different incident wavelengths change the overall intensity of the emission. In other words, the color of the phosphor material is regardless of the incident light. The relationship between the emission intensity and the excitation wavelength is described by the excitation spectrum. Fig. 4 shows an example of a phosphor

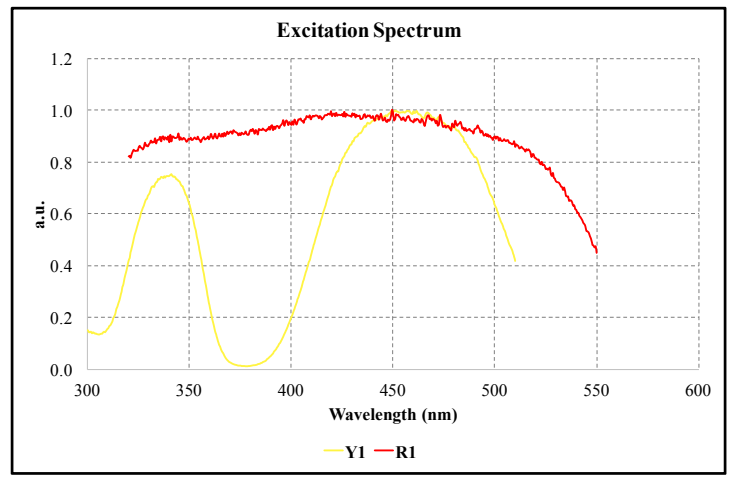

Fig. 2 Excitation Spectra of Yellow and Red Phosphors

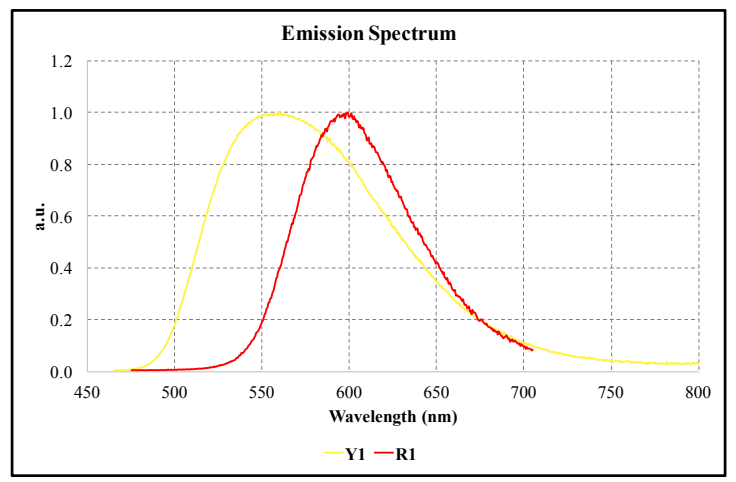

Fig. 3 Emission Spectra of Yellow and Red Phosphors

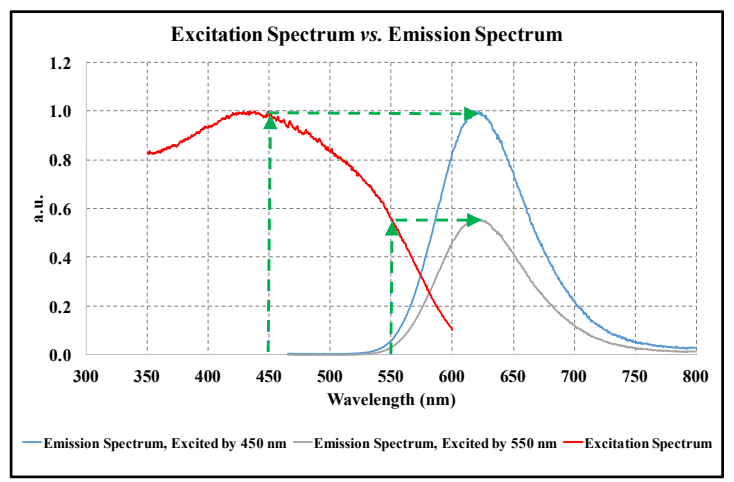

Fig. 4 Relationship between Excitation and Emission Spectra

material which gives the highest emission intensity when it is excited by $450 \mathrm{~nm}$ light. The emission intensity drops $42 \%$ when it is excited by $550 \mathrm{~nm}$ light. Nevertheless, the shape of the emission spectra remains the same in both cases.

\section{MiXed PHOSPHOR SPECTRUM CALCUATION}

As mentioned in the above section, the emission band of yellow phosphor is narrow. In order to improve the color rendering property of white LEDs, it is necessary to add more than one type of phosphor to broaden the emission spectrum. A small portion of red phosphor is commonly used to enhance the intensity in the red region. Since the host materials and dopants of red phosphors are different from that of yellow phosphors, the excitation spectra of red and yellow phosphors are dissimilar as shown in Fig. 2. Red phosphor has a wider excitation band. This wide excitation band may overlap with the emission spectrum of yellow phosphor. This means that the red phosphor is excited by 


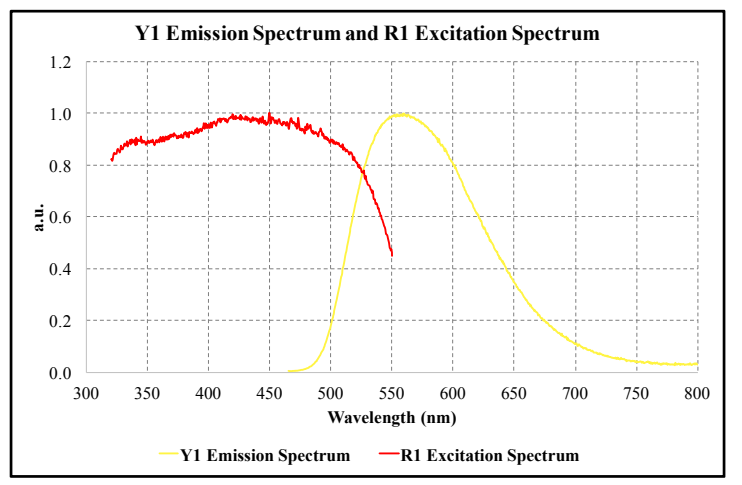

Fig. 5 Small Overlapping between Red Phosphor Excitation Spectrum and Yellow Phosphor Emission Spectrum

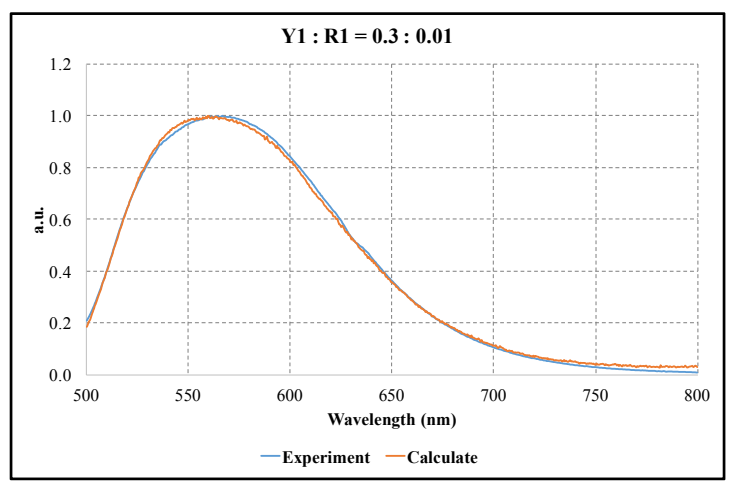

Fig. 6 Prediction Results of LED with Small Overlapping (by Eq. 1)

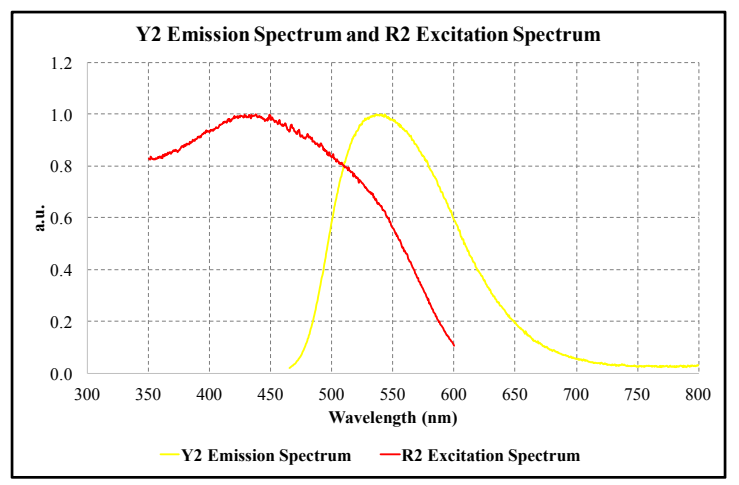

Fig. 7 Large Overlapping between Red Phosphor Excitation Spectrum and Yellow Phosphor Emission Spectrum

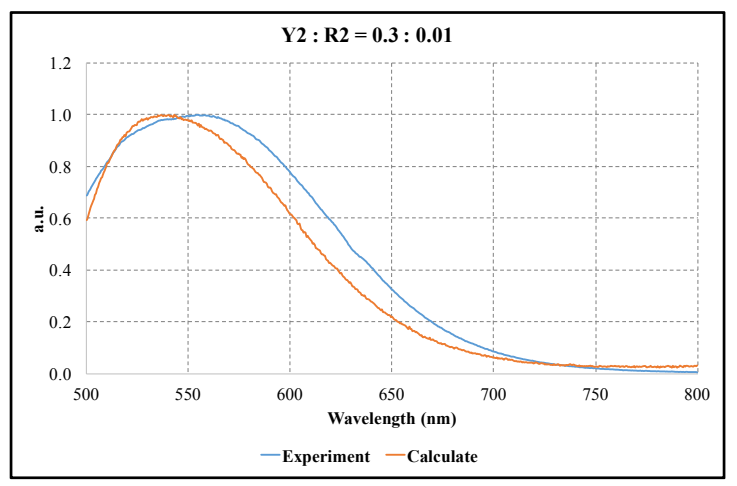

Fig. 8 Prediction Results of LED with Large Overlapping (by Eq. 1) both the blue light from the chip as well as the yellow light from the yellow phosphor. In other words, both blue and yellow light are absorbed by red phosphor.

Fig. 5 shows that there is a small overlapping between the $\mathrm{R} 1$ excitation spectrum and the $\mathrm{Y} 1$ emission spectrum at the 500 $\mathrm{nm}-550 \mathrm{~nm}$ range. If the yellow light absorption by the red phosphor is neglected, the final emission spectrum of the multiple phosphors can be estimated by a weighted average method as shown in Eq. 1.

$$
S_{\text {mix }}(\lambda)=Q Y_{Y} \cdot W_{Y} \cdot E m_{Y}(\lambda)+Q Y_{R} \cdot W_{R} \cdot E m_{R}(\lambda)
$$

where $S_{m i x}(\lambda)$ is the predicted spectrum of the mixed phosphor; $Q Y_{Y}$ and $Q Y_{R}$ are the quantum yields of yellow and red phosphor respectively; $W_{Y}$ and $W_{R}$ are the weight ratios of yellow and red phosphor respectively; $\operatorname{Em}_{Y}(\lambda)$ and $\operatorname{Em}_{R}(\lambda)$ are the emission spectra of yellow and red phosphor respectively.

Eq. 1 assumes the yellow and red phosphors share the same particle size distribution and are uniformly mixed together. Both yellow and red phosphor particles are excited by the same blue light intensity from the LED chip. Yellow light absorption by red phosphor is neglected and the emissions from yellow and red phosphors are independent. In light of the above, the final emission spectrum is a superposition of yellow and red emission spectra. The weights of the yellow and red emissions are directly proportional to the mixing weight ratio and the corresponding quantum yield of the phosphors.

A white light LED package with Y1 and R1 phosphors is fabricated. Y1 and R1 are uniformly mixed in a weight ratio of $0.3: 0.01$ to form a phosphor slurry. The slurry is dispensed into the reflective cup of a 5730 package. The spectrum of the LED package is measured and compared with that calculated by Eq. 1. Fig. 6 shows the normalized emission spectra in the $500 \mathrm{~nm}-$ $780 \mathrm{~nm}$ range. The result shows that the calculated emission spectrum matches well with the experimental results.

The overlapping of the red phosphor excitation spectrum and yellow phosphor emission spectrum is not always small. Fig. 7 shows that there is a large overlapping of the two at the $550 \mathrm{~nm}$ $-600 \mathrm{~nm}$ range when $\mathrm{Y} 2$ and $\mathrm{R} 2$ are used. This indicates that a considerable amount of $550 \mathrm{~nm}-600 \mathrm{~nm}$ light emitted from yellow phosphor is absorbed by red phosphor. The reabsorbed light is turned into red light by red phosphor subsequently.

Another white LED package with Y2 and R2 phosphors is fabricated by the same procedure as mentioned above. The mixing weight ratio applied also remains at $0.3: 0.01$. Fig. 8 compares the prediction result of Eq. 1 with the experimental result. There is an obvious difference between the prediction and experimental results, of which the prediction and experiment peak emission wavelengths are not the same. The prediction spectrum gives a higher intensity in the $500 \mathrm{~nm}-550 \mathrm{~nm}$ range and a lower intensity in the $550 \mathrm{~nm}-780 \mathrm{~nm}$ range. It is because Eq. 1 does not take the reabsorption and reemission by red phosphor into consideration. As a result, the model overestimates the yellow light intensity while at the same time underestimates the red light intensity.

The amount of yellow light reabsorbed by red phosphor is calculated by Eq. 2. The equation can be separated into three terms, i.e. $C, Q Y_{Y} \cdot W_{Y} \cdot E_{Y}(\lambda)$ and $\operatorname{Ex}_{R}(\lambda) . Q Y_{Y} \cdot W_{Y} \cdot E_{Y}(\lambda)$ 


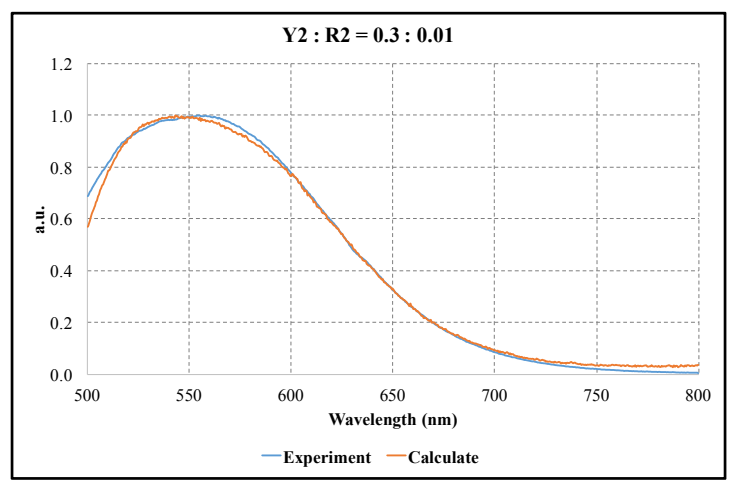

Fig. 9 Prediction Results of LED with Large Overlapping (by Eq. 4)

represents the total amount of yellow light emitted from yellow phosphor which is excited by the blue light from the LED chip. A certain amount of yellow light will reach the red phosphor particles and the corresponding ratio is defined by a numerical constant, $C$. The value of $C$ should be between 0 and 1 . Hence, $C \cdot Q Y_{Y} \cdot W_{Y} \cdot \operatorname{Em}_{Y}(\lambda)$ represents the intensity of yellow light on the red phosphor particles. A portion of yellow light is absorbed by red phosphor. The absorption spectrum of red phosphor describes the absorption behavior. As mentioned in the previous section, the excitation spectrum also includes phosphor absorption information. For simplicity, the normalized excitation spectrum of red phosphor, $\operatorname{Ex}(\lambda)$, is used to represent the absorption behavior. The amount of yellow light being absorbed by the red phosphor materials is calculated by the above three terms.

$$
R_{a b s}(\lambda)=C \cdot Q Y_{Y} \cdot W_{Y} \cdot E m_{Y}(\lambda) \cdot E x_{R}(\lambda)
$$

where $R_{a b s}(\lambda)$ is the reabsorption of yellow light by red phosphor; Rem is the reemission of red light; and $C$ is a numerical constant.

As mentioned in the previous section, the emission spectrum is controlled by the energy band gap of the phosphor material and is independent of the incident wavelength. The red phosphor emits the same color regardless of whether the incident light source is the blue LED chip or the yellow phosphor. Therefore, only the normalized emission spectrum of red phosphor is required to calculate the remission phenomenon. The intensity of the reemission is directly related to the total amount of yellow light being absorbed. The red light reemission is calculated by Eq. 3.

$$
R_{\text {em }}(\lambda)=Q Y_{R} \cdot W_{R} \cdot \sum R_{a b s} \cdot E m_{R}(\lambda)
$$

where $R_{e m}(\lambda)$ is the reemission of red phosphor.

The final prediction model is shown in Eq. 4. It considers the emissions of both yellow and red phosphors due to blue light excitation, reabsorption of yellow light by red phosphor and reemission of red light.

$$
\begin{aligned}
S_{\text {mix }}(\lambda)= & Q Y_{Y} \cdot W_{Y} \cdot E_{Y}(\lambda)+Q Y_{R} \cdot W_{R} \cdot E m_{R}(\lambda)- \\
& R_{a b s}(\lambda)+R_{e m}(\lambda)
\end{aligned}
$$

Fig. 9 compares the prediction result with the emission spectrum of the white light LED with Y $2+\mathrm{R} 2$ phosphors. It is noted that the calculated spectrum with a $C$ value of 0.15 gives the best match. The $x y$ chromaticity coordinates of the white
LED package and the prediction result are calculated. The emission from the blue LED chip is not considered in the calculation. Only the spectrum which is related to the phosphor emission, i.e. in the range of $500 \mathrm{~nm}-780 \mathrm{~nm}$, is used to calculate the chromaticity coordinate. The $x y$ coordinates of the white LED package and the prediction result are $(0.4260$, $0.5504)$ and $(0.4264,0.5509)$ respectively. The error is with in $+/-0.003$.

\section{CONCLUDING REMARKS}

Multiple phosphors are usually applied in white LEDs to enhance the color rendering performance. It is difficult to predict the emission spectrum accurately. The broad excitation spectrum of red phosphor usually overlaps with the yellow phosphor emission spectrum. Red phosphor is not only excited by blue light but also by a portion of yellow light emitted by yellow phosphor. A numerical model which has taken the phosphor reabsorption and reemission phenomenon into account is proposed in this paper. White light LEDs with multiple phosphors are fabricated. It is noted that the spectrum calculated by the proposed model matches perfectly with the experimental result. The error in CIE $x y$ coordinates is within $+/-0.003$.

\section{ACKNOWLEDGMENT}

This work was supported in part by a grant (T23-612/12-R) from the Research Council of the Hong Kong Special Administrative Region Government under the Theme-Based Research Scheme, and by the Hong Kong's University Grant Committee via the Area of Excellence project AoE-P04-08. This study was also supported by the Guangdong Provincial Department of Science and Technology (GDSTC) through two projects "Grant No. 2015B090901010"and "Grant No. 2014B050504001". The authors wish to acknowledge these support.

\section{REFERENCES}

[1] Y. Ohno, "Spectral design considerations for white LED color rendering," Optical Engineering, 44(11), 111302, November, 2005.

[2] Commission Internationale de I'Elcriage, Method of measuring and specifying colour rendering properties of light source, $3^{\text {rd }}$ edition, Vienna, Austria, 1995.

[3] W. Davis, and Y. Ohno, "Color quality scale," Optical Engineering, 49(3), 033602, 2010.

[4] IES Method for Evaluating Light Source Color Rendition, IES TM-30-15, 2015

[5] Commission Internationale de I'Elcirage, "Colorimetry," $3^{\text {rd }}$ edition, CIE 15: Technical Report, 2004

[6] H. Liu, J. C. C. Lo., and S. W. R. Lee, "Waffle Pack LED Module Configured Light Bulb and Simulation Validation," Proc. 10th China International Forum on Solid State Lighting (ChinaSSL 2013), 10 -12 Nov 2013, Beijing, China, pp.186.

[7] H. Liu, J. C. C. Lo, S. W. R. Lee, and H. Zhao, "LED Wafer Level Packaging with a Hemispherical Waffle Pack Remote Phosphor Layer," Proc. International Conference on Electronics Packaging (ICEP 2013), 10-12 Apr 2013, Osaka, Japan, , pp. 368-373.

[8] S. W. R. Lee, X. Guo, D. Niu and J. C. C. Lo, "Quasi-Conformal Phosphor Dispensing on LED for White Light Illumination," Proc. $63^{\text {rd }}$ Electronic Components \& Technology Conference (ECTC), 28- 31 May, 2013, Las Vegas, Nevada USA, pp. 563-567.

[9] J. C. C. Lo, H. Liu, S. W. R. Lee, X. Guo, and H. Zhao, "Remote Phosphor Deposition on LED Arrays with Pre-encapsulated Silicone Lens," Proc. EuroSimE 2013, 14-17 Apr 2013, Wroclaw, Poland. 
[10] Z. Tian, J. C. C. Lo, S. W. R. Lee, F. Yun, and R. Sun, "Investigation of the Influence of Ag Reflective Layer on the Correlated Color Temperature and the Angular Color Uniformity of LED with Conformal Phosphor Coating," Proc. 16th International Conference on Electronic Packaging Technology (ICEPT 2015), 11 - 14 Aug 2015, Changsha, China.

[11] N. Kimura, K. Sakuma, S. Hirafune, and K. Asano, "Extrahigh color rendering white light-emitting diode lamps using oxynitride and nitride phosphors excited by blue light-emitting diode," Applied Physics Letters, vol. 90, 2007, 051109.

[12] H. Wu, X. Zhang, C. Guo, J. Xu, M. Wu, and Q. Su, "Three-band white light from InGaN-based blue LED chip precoated with green/red phosphors," IEEE Photonics Technology Letters, vol. 17, no. 6, June 2005, pp. 1160-1162.

[13] L. Chen, C. I. Chu, and R. S. Liu, "Improvement of emission efficiency and color rendering of high-power LED by controlling size of phosphor particles and utilization of different phosphors," Microelectronics Reliability, vol. 52, 2012, pp. 900-904.

[14] M. Zheng, W. Ding, F. Yun, D. Xia, Y. Huang, Y. Zhao, W. Zhang, M. Zhang, M. Guo, and Y. Zhang, "Study of high CRI white light-emitting diode devices with multi-chromatic phosphor," Proc. $64^{\text {th }}$ IEEE Electronic Components and Technology Conference (ECTC 2014), Orlando, FL, USA, 27 May - 30 May 2014, pp. 2236-2240.

[15] T. Fukui, K. Kamon, J. Takeshita, H. Hayashi, T. Miyachi, Y. Uchida, S. Kurai, and T. Taguchi, "Superior illuminant characteristics of color rendering and luminous efficacy in multilayered phosphor conversion white light sources excited by near-ultraviolet light-emitting diodes," Japanese Journal of Applied Physics, vol. 48, 2009, 112101.

[16] G. He, and H. Yan, "Optimal spectra of the phosphor-coated white LEDs with excellent color rendering property and high luminous efficacy of radiation," Optics Express, vol. 19, no. 3, 2011, pp. 2519-2529.

[17] C. C. Lin and R. S. Liu, "Advanced in phosphors for light-emitting didoes," Journal of Physical Chemistry Letters, vol. 2, 2011, pp. 12681277.

[18] J. C. C. Lo, S. W. R. Lee, X. Guo and H. Zhao, "Multiplayer Dispensing of Remote Phosphor for LED Wafer Level Packaging with Pre-formed Silicone Lens," Proc. 5th Electronics System-Integration Technology Conference, 16 - 18 Sep 2014, Helsinki, Finland.

[19] J. Wang, J. C. C. Lo, S. W. R. Lee, F. Yun and M. Tao, "Modeling and Parametric Study of Light Scattering, Absorption and Emission of Phosphor in a White Light-Emitting Diode," Proc. InterPACKICNMM 2015, 6 - 9 Jul 2015, San Francisco, California, USA.

[20] C. C. Sun, C. Y. Chen, J. H. Chang, T. H. Yang, W. S. Ji, Y. S. Jeng, and H. M. Wu, "Linear calculation model for prediction of color rendering index performance associated with correlated color temperature of white light-emitting diodes with two phosphors," Optical Engineering, vol. 51, 054003, May 2012. 